\title{
HAMILTON CYCLES IN DOUBLE GENERALIZED PETERSEN GRAPHS
}

\author{
Yutaro SAKAMoto \\ Department of Informatics \\ The University of Electro-Communications, Japan \\ e-mail: y-sakamoto@uec.ac.jp
}

\begin{abstract}
Coxeter referred to generalizing the Petersen graph. Zhou and Feng modified the graphs and introduced the double generalized Petersen graphs (DGPGs). Kutnar and Petecki proved that DGPGs are Hamiltonian in special cases and conjectured that all DGPGs are Hamiltonian. In this paper, we prove the conjecture by constructing Hamilton cycles in any given DGPG.
\end{abstract}

Keywords: double generalized Petersen graph, Hamilton cycle.

2010 Mathematics Subject Classification: 05C45, 05C38.

\section{REFERENCES}

[1] B. Alspach, The classification of Hamiltonian generalized Petersen graphs, J. Combin. Theory Ser. B 34 (1983) 293-312. doi:10.1016/0095-8956(83)90042-4

[2] F. Castagna and G. Prins, Every generalized Petersen graph has a Tait coloring, Pacific J. Math. 40 (1972) 53-58. doi:10.2140/pjm.1972.40.53

[3] H.S.M. Coxeter, Self-dual configurations and regular graphs, Bull. Amer. Math. Soc. 56 (1950) 413-455. doi:10.1090/S0002-9904-1950-09407-5

[4] X. Fu, Y. Yang and B. Jiang, On the domination number of generalized Petersen graphs $P(n, 2)$, Discrete Math. 309 (2009) 2445-2451. doi:10.1016/j.disc.2008.05.026

[5] R.M. Karp, Reducibility among combinatorial problems in: R.E. Miller and J.W. Thatcher (Eds.), (Plenum Press, New York, 1972) 85-103 doi:10.1007/978-1-4684-2001-2_9. 
[6] K. Kutnar and P. Petecki, On automorphisms and structural properties of double generalized Petersen graphs, Discrete Math. 339 (2016) 2861-2870. doi:10.1016/j.disc.2016.05.032

[7] M.E. Watkins, A theorem on Tait colorings with an application to the generalized Petersen graphs, J. Combin. Theory 6 (1969) 152-164. doi:10.1016/S0021-9800(69)80116-X

[8] J.-X. Zhou and Y.-Q. Feng, Cubic vertex-transitive non-Cayley graphs of order $8 p$, Electron. J. Combin. 19 (2012) \#P53.

[9] J.-X. Zhou and Y.-Q. Feng, Cubic bi-Cayley graphs over Abelian groups, European J. Combin. 36 (2014) 679-693. doi:10.1016/j.ejc.2013.10.005

Received 25 November 2016

Revised 10 June 2017

Accepted 10 June 2017 